\title{
Derivaciones digestivas guiadas por ultrasonido endoscópico
}

\author{
Gustavo López-Arce Ángeles* \\ Departamento de Endoscopia, Hospital Juárez de México, Ciudad de México, México
}

\section{Resumen}

Recientemente se han descrito técnicas endoscópicas que son una alternativa para el manejo de pacientes que requieren manejo paliativo para obstrucción a la vaciamiento gástrica o para pacientes que requieren CPRE con anatomía modificada, se han realizado fístulas gastroentéricas y enteroentéricas guiadas por USE y colocación de LAMS. En varios trabajos presentados en la DDW 2020 y la UEGW 2019, los resultados en cuanto al éxito técnico y clínico en la mayoría de los estudios son superiores al 90\%, una de las complicaciones es el desplazamiento de la prótesis, por lo que algunos autores recomiendan realizar la fístula en un primer momento y 2 a 3 semanas realizar la CPRE, un estudio observó cuando la CPRE se realizó 3 días después de la fístula tenían menor desplazamiento. Las complicaciones ocurren en 15-20\% y las graves en $<5 \%$ en la mayoría de los estudios. Un estudio con seguimiento a 6 meses no observó eventos adversos a largo plazo y en los pacientes con bypass gástrico tampoco se observó ganancia de peso posterior. Al comparar la CPRE por enteroscopia de doble balón vs. EDGE, esta última mostró tendencia a mayor éxito técnico y fue efectiva para litos de $13 \mathrm{~mm}$, en la mitad del tiempo de procedimiento.

\section{Introducción}

Las derivaciones digestivas guiadas por ultrasonido endoscópico son una técnica relativamente nueva en el área de endoscopia terapéutica, el primer reporte que se tiene es en 1991 por Swain, et al ${ }^{1}$ en animales, en el cual se realizó por medio de laparoscopia y endoscopia una anastomosis enteral, posterior a ello se presentaron varios reportes de casos de procedimientos y NOTES (Natural Orifice Transluminal Endoscopic Surgery) ${ }^{2,3}$, fue hasta 2012 que Binmoeller ${ }^{4}$ en modelos porcinos realizó anastomosis con prótesis de aposición luminal (LAMS por sus siglas en inglés) a partir de ese entonces se han realizado varios estudios en humanos. Las utilidades de la derivaciones biliodigestivas guiadas por USE principalmente se enfocan en pacientes con anatomía modificada por cirugía en pacientes que requieren acceso a la vía biliar o ámpula de Vater, como medida paliativa en pacientes con obstrucción de salida gástrica (GOO) benigna o maligna y como tratamiento en pacientes con síndrome de asa aferente (AFS). En cuanto al tratamiento de obstrucción gástrica se han realizado diferentes técnicas, las dos principales es con ayuda de un balón para facilitar la localización del asa yeyunal y por otro lado la técnica con punción directa del asa a través del estómago, en este sentido en la DDW 2020 destaca un trabajo realizado por el grupo de Ali FS et al. ${ }^{5}$ compararon ambas técnicas en un metaanálisis que incluyeron 218 pacientes y encontraron que el éxito técnico fue similar en ambas técnicas $93 \%$ y $96 \%$ respectivamente, tampoco hubo diferencias en el éxito clínico (100\% vs. $98 \%$ ) ni en la incidencia de complicaciones, sin embargo el tiempo 
del procedimiento fue el doble en el grupo asistido con balón (43 vs. 88 minutos), en el trabajo no se establece si estos resultados tuvieron significancia estadística. Una de las interrogantes radica en el calibre de las LAMS, los primeros reportes se realizaron con LAMS de $15 \mathrm{~mm}$, Sobani ZA et al. ${ }^{6}$ valoraron la eficacia, seguridad y éxito técnico y clínico en un estudio retrospectivo con LAMS con cauterio integrado (Hot AXIOS, Boston Scientific) de $20 \mathrm{~mm}$ en 13 pacientes con GOO, el éxito técnico fue del $100 \%$ y en el seguimiento a 59 \pm 45 días solamente un paciente requirió reintervención con dilatación hidroneumática de la prótesis por oclusión, con lo que se concluye que es probable que las prótesis de $20 \mathrm{~mm}$ permanezcan más tiempo permeables pero es necesario comparar ambos calibres en estudios prospectivos. Masarik $\mathrm{V}$, et al. reportaron 33 procedimientos en 32 pacientes con GOO $(n=16)$ y AFS $(n=17)$, el éxito técnico fue en 29 pacientes y 3 pacientes requirieron tratamiento quirúrgico por falla en la colocación de LAMS, los eventos adversos se presentaron en $6(18.2 \%)$ pacientes y un paciente murió por hemorragia al 4 día del procedimiento, todos los pacientes tratados por AFS ( $n=16 / 100 \%$ ) tuvieron éxito clínico. Se comparó en un estudio retrospectivo el tratamiento de gastroyeyunostomía por endoscopia vs. el tratamiento quirúrgico en 52 pacientes con carcinomatosis ${ }^{7}$ no se encontraron diferencias en el éxito clínico o técnico, recurrencia de oclusión de la anastomosis o mortalidad, aunque las LAMS que se utilizaron fueron de 10 y $15 \mathrm{~mm}$. Wannhoff A. et al. ${ }^{8}$ encontraron que el problema técnico más frecuente fue el desplazamiento de la copa distal (57.1\%), el éxito clínico fue de $74 \%$ y los eventos adversos $14.3 \%$. La distancia entre los dos tejidos ( $9 \mathrm{~mm}$ vs. $20 \mathrm{~mm} ; \mathrm{p}<0.004$ ) fue un predictor de éxito técnico.

Se comparó el éxito técnico y clínico de CPRE transgástrica directa por ultrasonido endoscópico (EDGE) vs. CPRE con doble balón (DBE-ERCP) en un estudio retrospectivo ${ }^{9}$ con (72 procedimientos $/ 41$ pacientes), no hubo diferencias significativas en el éxito terapéutico ( $100 \%$ vs. $84 \%$; $p=0.06)$, sin embargo en el grupo de EDGE los litos eran más grandes (13.5 mm vs. 8.9 $\mathrm{mm} ; \mathrm{p}<0.001$ ), el tiempo de procedimiento fue menor en EDGE (49 vs. $67 \mathrm{~min} ; \mathrm{p}<0.04$ ), ni diferencias significativas en eventos adversos ( $11 \%$ vs. $6 \%$; $p=0.53$ ) y en todos los pacientes de EDGE se observó cierre espontáneo de la fístula 14 días después, los autores concluyen que EDGE podría ser de mayo utilidad en litos grandes en pacientes con anatomía modificada. En los casos de bypass gástrico una preocupación es la posibilidad de que la fístula gastrogástrica pueda persistir y con ello provocar ganancia en peso, en un estudio prospectivo ${ }^{10}$ se evaluaron 17 pacientes con bypass gástrico y no se observó ganancia de peso en ninguno de los pacientes en el seguimiento a $6 \pm 2$ meses, además de que en el $81 \%$ se removió la LAMS posterior en la misma intervención, uno de los eventos adversos es la migración de la LAMS, por lo que varios grupos sugieren realizar primero la gastro-gastroanastomosis y de 2 a 3 semanas realizar la CPRE, sin embargo no está bien establecido el tiempo, en un trabajo multicéntrico retrospectivo ${ }^{11}$ se encontró que la migración de los LAMS cuando se realiza en una misma sesión la CPRE se presenta menos con LAMS de 20 mm vs. 15 mm (RM 7.9 [1.3-47]; $p=0.02$ ), también la migración fue menor cuando se fijó con sutura endoscópica $(p=0.032)$ en los que se dilató la prótesis $(p=0.026)$ y hubo tendencia sin significancia estadística cuando el acceso fue transgástrico vs. enteral (10/91 vs. $2 / 40 ; p=0.34$ ) de los pacientes que presentaron migración (3 requirieron cirugía, 2 colocación de prótesis esofágica y 2 un segundo LAMS) 2 pacientes presentaron hemorragia en el momento del procedimiento que fue manejada por endoscopia en la misma intervención, en ese sentido se presentaron los resultados de 19 pacientes a los cuales se le realizó EDGE con LAMS de $20 \mathrm{~mm}$, a 10 pacientes se les realizó en el misma sesión y al resto 3 días después, el primer grupo tuvo mayor migración de la LAMS (RM 8.41), en este estudio no se especifica si se realizó dilatación de la prótesis, no se observaron diferencias significativas en el éxito técnico el cual fue de $90 \%$ y $100 \%$ respectivamente ${ }^{12}$. En un estudio multicéntrico ${ }^{13}$ se evaluaron las complicaciones a largo plazo de EDGE, se incluyeron 166 pacientes y se siguieron por 5.7 meses, de estos el $51 \%(n=85)$ se realizó en la misma sesión y el resto en dos $(n=81)$ y el $52 \%$ se realizó por vía transgástrica, el tiempo medio del procedimiento fue de 87 minutos, solamente en el $21 \%$ se fijó el LAMS a la mucosa con suturas, hemoclips o prótesis doble cola de cochino. Las complicaciones periprocedimiento se presentaron en $17 \%$ (leve $3.1 \%$, moderadas $11.5 \%$ y graves $2.4 \%$ ) los 4 pacientes con complicaciones graves fueron manejados por laparoscopia, se retiró la prótesis en una media de 47 días, en $27 \%$ no se realizó cierre endoscópico del trayecto fistuloso y de manera global en $12 \%$ tuvo persistencia de la fístula, se requirió una media de $1.2 \pm 0.8$ procedimientos endoscópicos para cerrar la misma, lo que resultó efectivo en el $71 \%$, la DM2 se asoció con un factor de persistencia de la fístula (RM 7.2 [1.5 34]) Finalmente un metaanálisis evaluó 290 pacientes 
(62\% por GOO y EDGE $27 \%$ ), el éxito técnico global y clínico fue de $93 \%$ y $90 \%$ respectivamente, los eventos adversos en $11.7 \%$ y de estos los eventos adversos fatales fueron del $2.9 \%{ }^{14}$. Otro estudio coincide con el éxito técnico en $100 \%$, reportó el cierre espontáneo de las fístulas en $72 \%$ y hemorragia posterior al procedimiento en $6 \%{ }^{15}$.

\section{Conflicto de intereses}

Los autores declaran no tener conflicto de intereses alguno.

\section{Financiamiento}

Los autores no recibieron patrocinio para llevar a cabo este artículo.

\section{Bibliografía}

1. Swain CP, Mills TN. Anastomosis at flexible endoscopy: an experimental study of compression button gastrojejunostomy. Gastrointest Endosc. 1991;37(6):628-31.

2. Kantsevoy SV, Jagannath SB, Niiyama H, Chung SS, Cotton PB, Gostout CJ, et al. Endoscopic gastrojejunostomy with survival in a porcine model. Gastrointest Endosc. 2005;62(2):287-92.

3. Bergstrom M, Ikeda K, Swain P, Park PO. Transgastric anastomosis by using flexible endoscopy in a porcine model (with video). Gastrointest Endosc. 2006;63(2):307-12.
4. Binmoeller KF, Shah JN. Endoscopic ultrasound-guided gastroenterostomy using novel tools designed for transluminal therapy: a porcine study. Endoscopy. 2012;44(5):499-503.

5. Ali FS, Soin S, Hussain M, et al.Endoscopic ultrasound-guided gastroenterostomy for the treatment of gastric outlet obstruction; a meta-analysis of direct and balloon-assisted approach. DDW 2020. Resumen 1248.

6. Sobani ZA, Rustagi T. Endoscopic ultrasound guided gastroenterostomy using large (20 mm) diameter lumen apposing metal stents. DDW 2020. Resumen 1330.

7. Bondi G, Bazarbashi AN, Abbas AM. et al. endoscopic gastroenterostomy versus surgical gastrojejunostomy for the treatment of gastric outlet obstruction in patients with peritoneal carcinomatosis: a retrospective comparative study. DDW 2020. Resumen 1269.

8. Wannhoff A, Ruh N, Meier B, et al. Predictors of technical and clinical success of endoscopic gastroenterostomy with lumen apposing metals stents. DDW 2020. Resumen 1331

9. Abidali H, Rezaie A, Omer A, et al. EUS-directed transgastric ERCP versus double balloon enteroscopy-assisted ERCP in patients with roux-en-y gastric bypass anatomy: a single center experience. DDW 2020. Resumen 1427.

10. Nguyen NA, Nett A, Hamerski CM et al. Impact of EUS guided gastro-gastrostomy to facilitate further endoscopic interventions in patients with rouxen-y gastric bypass: more than just EDGE!. DDW 2020. Resumen 1425.

11. Shinn B, Boortalary T, Raijman I et al. Maximizing success in single-session edge - predictive factors of stent migration. DDW 2020 Resumen 960.

12. Krafft MR, Shah-Khan SM, Fang $W$, et al. «AHHH, this interval is just right!" the story of edge and the three intervals. can a shortened (several day) interval between eus-gastrogastrostomy and transgastric ercp mitigate the risk of stent (LAMS) dislodgment while facilitating a timely ERCP? DDW 2020. Resumen 1317.

13. Runge T, Kowalski T, Baron TH et al. Living on the EDGE: Long-Term Complications, and Implications Following EUS-Directed Transgastric ERCP (EDGE): A Multicenter Study. UEG Week 2019, United European Gastroenterology Journal Volume 7 Issue 8 (Supplement), October 2019.

14. Antonelli G, Kovacevic B, Karstensen JG et al. Endoscopic ultrasound-guided gastro-enteric anastomosis: A systematic review and meta-analysis. UEG Week 2019, United European Gastroenterology Journal Volume 7 Issue 8 (Supplement), October 2019.

15. Mohy-ud-din N, Carleton N, Syed A. et al. Endoscopic Ultrasound (EUS)-Directed Transgastric Endoscopic Retrograde Cholangiopancreatography (ERCP): A Single Center Experience of An Evolving Procedure. UEG Week 2019, United European Gastroenterology Journal Volume 7 Issue 8 (Supplement), October 2019. 